\title{
Análisis de un generador de HHO de celda seca para su aplicación en motores de combustión interna
}

\section{Analysis of a dry cell HHO generator for application in internal combustion engines}

\author{
Ángel Orlando Díaz-Rey ${ }^{1}$, Jonathan Eduardo González-Gil², Octavio Andrés González-Estrada ${ }^{3}$ \\ ${ }^{1}$ INMECSOL S.A.S., Floridablanca, Colombia, Email: inmecsol@gmail.com \\ ${ }^{2}$ Agropecuaria Galeano Rueda y Cía S.A.S., Acacías, Colombia, Email: jhonatan021@ @otmail.com \\ ${ }^{3}$ Grupo de Investigación en Energía y Medio Ambiente-GIEMA, Escuela de Ingeniería Mecánica, Universidad Industrial de \\ Santander, Ciudad Universitaria, Bucaramanga, Colombia. Orcid: 0000-0002-2778-3389, Email: agonzale@ saber.uis.edu.co
}

RECIBIDO: Abril 27, 2017. ACEPTADO: Junio 14, 2017. Versión FinAL: Octubre 25, 2017.

\section{RESUMEN}

Los motores de combustión interna a gasolina tienen conocidos problemas de eficiencia térmica y alto índice de emisión de gases contaminantes. Por otro lado, existen procesos químicos y eléctricos que han permitido obtener fuentes de energía limpia del agua. La electrólisis permite mediante un proceso electroquímico la obtención de gas de hidrógeno y oxígeno, de cuya mezcla se obtiene HHO. Las características moleculares del HHO permiten la disminución de gases tóxicos al ambiente y el ahorro de combustibles hidrocarbonados en motores de combustión interna. En este trabajo se investigan los beneficios potenciales de la mezcla de HHO como aditivo a los combustibles fósiles, en particular, la gasolina comercial. Se describe el diseño de un sistema con una celda electrolítica y se evalúa el desempeño de un motor de $110 \mathrm{~cm}^{3}$ en términos del consumo específico de combustible, potencia, torque y emisión de gases.

PALABRAS ClAVE: electrólisis; celdas de oxihidrógeno; motores de combustión interna; energía limpia; combustibles.

\begin{abstract}
Gasoline internal combustion engines have known issues related to thermal efficiency and high emission of polluting gases. On the other hand, there are chemical and electrical processes that have provided sources of clean energy from water. Electrolysis allows the production of hydrogen and oxygen gas by means of an electrochemical process, from which HHO is obtained. The molecular characteristics of HHO allow the reduction of toxic gases to the environment and the saving of hydrocarbon fuels in internal combustion engines. In this paper, we investigate the potential benefits of HHO mixtures as an additive to fossil fuels, in particular, commercial gasoline. We describe the design of a system with an electrolytic cell and evaluate the performance of a $110 \mathrm{~cm}^{3}$ engine in terms of brake specific fuel consumption, brake power, brake torque and gas emissions.
\end{abstract}

KEYWORDS: electrolysis; oxyhydrogen cells; internal combustion engines; clean energy; fuels. 


\section{INTRODUCCIÓN}

Hoy en día, uno de los principales retos de la ingeniería es reducir el consumo de combustibles para lograr, entre otras cosas, menores índices de emisiones de gases contaminantes provocados por los motores de combustión interna y utilizar fuentes renovables de energía [1]-[3]. La utilización de combustibles fósiles para la obtención de energía se ha traducido en un problema medioambiental que ha escalado rápidamente en todo el mundo. En particular, se buscan soluciones que no involucren alguna trasformación física en los motores. Una de las líneas de investigación de mayor interés comprende el uso de combustibles alternativos, que aumenten la eficiencia del motor y reduzcan la emisión de gases contaminantes [4]. El hidrógeno, como combustible alternativo, presenta dichas características, sin embargo, la manufactura de motores que integren el hidrógeno como combustible no es por ahora rentable desde el punto de vista comercial, ya que aumentan demasiado los costos de fabricación [5], [6].

Momirlan y Veziroglu [7] presentan una descripción de la reciente transición del uso de combustibles fósiles a energías renovables, i.e. hidrógeno como fuente de limpia de energía. Se reportan investigaciones sobre los beneficios potenciales de la tecnología, usos actuales, métodos de producción y almacenamiento, y los retos que afronta la tecnología: costo, infraestructura para el transporte y eficiencia en la producción.

Saravanan et al. [8], [9] investigaron experimentalmente la mezcla aire-hidrógeno en motores diésel, mejorando la eficiencia hasta un $27,9 \%$, con una carga constante, esto mediante la adición de un $30 \%$ de hidrógeno. También se observó que el consumo de combustible se redujo con el incremento del porcentaje de hidrógeno. Se probó la inyección de varios caudales de hidrógeno en el colector optimizado de un motor diésel, mostrando que la mejor condición de operación de la inyección de hidrógeno era iniciarla en el punto muerto superior (PMS), manteniéndola por $30^{\circ}$ y con un flujo de $7.5 \mathrm{l} / \mathrm{min}$. Saravanan et al. [10] realizaron estudios experimentales sobre el hidrógeno como complemento en motores diésel usando la técnica de recirculación de gases de escape. Los resultados arrojaron que el consumo específico de combustible se redujo sin la necesidad de utilizar la técnica de recirculación de gases al aplicar un flujo de 20 $\mathrm{L} / \mathrm{min}$ de hidrógeno. Entre las razones por la cuales el hidrógeno es una buena opción para motores diésel, está que podrían bajar los niveles de heterogeneidad debido a la alta difusividad del hidrógeno, haciendo la mezcla más uniforme con el premezclado con aire, a esto también se le suma la alta velocidad de propagación de la llama en comparación con otros combustibles [11].
Masood et al. [12] realizaron modelos numéricos de la combustión de diferentes mezclas de hidrógeno-diésel, verificando los resultados con pruebas experimentales. Se utilizaron porcentajes de hidrógeno entre el 20 y $80 \%$. Los autores concluyeron que las mezclas resuelven el problema del funcionamiento pobre de combustibles hidrocarbonados, p.e., diésel puro, el cual presenta dificultad para encender, disminuyendo la potencia, rendimiento y economía del combustible.

Baltacioglu et al. [13] estudiaron la inyección de mezclas de diferentes combustibles alternativos con biodiesel para mirar los efectos en el rendimiento y en la emisión de gases contaminantes. Las pruebas se realizaron enriqueciendo la mezcla de biodiesel con un $10 \%$ de diésel, y el aire con hidrógeno puro o HHO. La cantidad de hidrógeno y HHO se ajustó a $10 \mathrm{l} / \mathrm{min}$ en un suministro constante. Los resultados se evaluaron de acuerdo con los parámetros de rendimiento del motor y las emisiones contaminantes, entre las 1200 y 2600 RPM, y se compararon los datos obtenidos con diésel estándar. Los resultados fueron buenos respecto a la disminución de gases contaminantes, no habiendo mucha diferencia entre la utilización de hidrógeno puro y el HHO.

Akansu et al. [14] presentaron una revisión del uso de mezclas de gas natural - hidrógeno en motores de combustión interna. Los autores indican que el uso de convertidores catalíticos disminuye la emisión de gases contaminantes a niveles extremadamente bajos, alcanzando niveles de estándares de vehículos de emisión cero (EZEV). Ma et al. [15] estudiaron las mezclas de hidrógeno con gas natural, y mostraron que se alcanzaban intervalos más cortos de propagación y desarrollo de la llama. Ji et al. [16] estudiaron los efectos del hidrógeno en un motor por inyección alimentado con metanol. Se obtuvieron altos niveles en la presión media efectiva al freno al igual que la eficiencia térmica y volumétrica, con valores aproximadamente del $3 \%$ de hidrógeno en la mezcla de admisión.

Dado que la ignición del hidrógeno se da con baja energía y se quema mucho mejor en la combustión, se podría decir que los motores que funcionan con hidrógeno son los más indicados para funcionamiento a condiciones más conservativas, lo que a su vez ayuda al ahorro económico y la emisión de gases contaminantes [17][18]. Musmar [19] y Al-Rousan [20] diseñaron dispositivos capaces de generar HHO para motores a gasolina. Los autores mostraron que los porcentajes de emisión de contaminantes se reducían hasta en un 50\% de óxidos de nitrógeno, $20 \%$ de monóxido de carbono y el gasto de combustible en un $30 \%$ al ser agregado a la mezcla de combustión gas HHO. Yilmaz et al. [21] mostraron 
aumentos de cerca del 19,1\% del par motor, además de la reducción de $\mathrm{CO}$ en un 13,5\%, emisiones de hidrocarburos en un $5 \% \mathrm{y}$, respecto al consumo específico de combustible, una reducción del $14 \%$.

Shivaprasad et al. [22] realizaron experimentos con motores con sistema de inyección de un solo cilindro, en donde agregaban valores de hidrógeno en fracciones volumétricas, en porcentajes entre el $5 \%$ y $25 \%$. Se determinó un aumento en la presión media efectiva al freno, al igual que en la eficiencia térmica. Por otra parte, se obtuvieron valores más bajos de hidrocarburos y monóxido de carbono, aunque los valores de óxidos de nitrógeno se incrementaron porcentualmente al adicionar hidrógeno. Wang et al. [23], [24] estudiaron el uso de mezclas de gas hidroxi (HHO) y de hidrógeno-oxígeno en motores de gasolina, mostrando mejores resultados para el segundo tipo de mezcla.

Wang et al. [24], [25] llevaron a cabo experimentos en un motor a gasolina para estudiar su rendimiento con la adición de hidrógeno, a condiciones normales de 1400 RPM. Los análisis en [26] demuestran una mejora en los valores de la presión media efectiva al freno y la eficiencia térmica a condiciones muy por debajo de las normales, como también un aumento en la presión del cilindro junto con un avance del ángulo del cigüeñal causado por el incremento porcentual de hidrógeno. La velocidad de llama de hidrógeno es superior, y al mezclarse con gasolina puede quemarse más rápido. Pero si el hidrógeno que se adiciona incrementa su proporción, y la cantidad de combustible se hace más pobre, la velocidad de reacción será menor y la combustión se realizará de manera deficiente. Por esta razón, Ji et al. [27] investigaron las consecuencias de la sincronía de la chispa en el proceso de combustión para un rendimiento térmico más alto y una presión efectiva media indicada.

En este trabajo se estudia la influencia de la adición de gas $\mathrm{HHO}$ en la mezcla de combustión de un motor de motocicleta de $110 \mathrm{~cm}^{3}$. En primer lugar, se presentan las generalidades de los motores de combustión interna y las características de las emisiones contaminantes. Se presenta una descripción del hidrógeno como combustible limpio. Luego, se explica el proceso básico de obtención del gas HHO y se describe el proceso de electrólisis. Posteriormente, se presenta el diseño del experimento y se muestra el esquema de montaje de la celda de gas HHO. Seguidamente, se realiza el análisis de resultados $\mathrm{y}$, finalmente, se presentan las conclusiones más relevantes.

\section{SISTEMA DE COMBUSTIÓN CON HHO}

Los motores de combustión interna son máquinas que obtienen energía mecánica a través del proceso químico de combustión, resultado de la quema de un combustible dentro de la cámara de combustión. En principio, el ciclo que rige la mayoría de motores de combustión interna es el denominado ciclo Otto, comúnmente llamado motor de cuatro tiempos, el cual precisa de cuatro carreras de pistón (dos vueltas completas de cigüeñal) para completar el ciclo termodinámico de su combustión. Estos cuatro tiempos se deben a la admisión, compresión, combustión, y escape [28].

En la admisión desciende el pistón absorbiendo la mezcla de aire y combustible. La válvula de escape permanece cerrada, mientras que la de admisión permanece abierta. En la etapa de compresión, la válvula de admisión se cierra en el momento en que termina el recorrido en la parte inferior, comprimiendo el gas contenido en la cámara al ascender el pistón. En la etapa de combustión, cuando llega al final de la carrera en la parte superior, la presión del gas es máxima, dando paso a la inflamación de la mezcla provocada por la chispa en la bujía. Una vez ha iniciado la combustión se obtiene un incremento de temperaturas y la presión dentro del cilindro, finalizando con la única etapa en la que se obtiene trabajo. En la última fase los gases son empujados por el pistón a través de la válvula de escape, y reiniciando el ciclo [29].

Algunas de las características de este ciclo de trabajo están relacionadas a la admisión de una mezcla homogénea de combustible, la mezcla se comprime y la combustión empieza tras el salto de la chispa a lo cual se le denomina MEP o encendido provocado. La regulación de la carga es cuantitativa, la variación del rango de cantidades de aire y combustible es mínima, pero a mayor demanda de potencia mayor es la cantidad de mezcla que se debe proporcionar [29].

\subsection{Composición de las emisiones de gases}

El uso de combustibles fósiles para el funcionamiento de motores de combustión interna produce la liberación de gases contaminantes que se convierten en un gran problema para el medio ambiente y que en un tiempo dado afecta la salud de las personas [1]. Por la forma en la que trabajan los motores de combustión interna, no queman la totalidad del combustible, y si a esto le sumamos el hecho de que su combustión no se controla correctamente, mayores serán los niveles de contaminación emitidos hacia la atmósfera. Se tienen dos grupos distintos de gases que se producen por la combustión: los no tóxicos y los tóxicos. En el primer grupo tenemos el nitrógeno, oxígeno, agua y dióxido de 
Tabla 1. Emisiones estándar de gases de combustión.

\begin{tabular}{|l|l|l|l|l|}
\hline & Carburación & $\begin{array}{l}\text { Inyección sin } \\
\text { catalizador }\end{array}$ & $\begin{array}{l}\text { Inyección antes del } \\
\text { catalizador }\end{array}$ & $\begin{array}{l}\text { Inyección después } \\
\text { del catalizador }\end{array}$ \\
\hline CO & Entre 1\% y 2\% & $1+-0,5 \%$ & Entre 0,4\% y 0,8\% & Menor de 0,2\% \\
\hline CO2 & Mayor de 11\% & Mayor de 12\% & Mayor de 13\% & Mayor de 13,5\% \\
\hline $\mathbf{H C}$ & Menor de 400 ppm & Menor de 300 ppm & Menor de 250 ppm & Menor de 100 ppm \\
\hline $\mathbf{O 2}$ & Menor de 3,5\% & Menor de 2,5\% & Menor de 1,5\% & Menor de 0,2\% \\
\hline $\boldsymbol{\lambda}$ & & & Entre 0,99 y 1,02 & Entre 0,99 y 1,01 \\
\hline RPM & & & Ralentí & 2000 RPM \\
\hline
\end{tabular}

carbono (CO2). Dentro del segundo grupo tenemos el monóxido de carbono ( $\mathrm{CO}$ ), dióxido de azufre, plomo, hidrocarburos (HC) y partículas de hollín.

En la Tabla 1 se muestran valores porcentuales de algunos de los gases de emisión con diferentes configuraciones del sistema de inyección [30].

\subsection{Hidrógeno como combustible alternativo}

En la búsqueda de opciones amigables con el ambiente, se ha investigado la utilidad del hidrógeno como combustible limpio para el reemplazo de los combustibles fósiles en motores de combustión interna. Se ha utilizado hidrógeno por sus cualidades tales como su alto número de octano (130), el cual mide la capacidad antidetonante del combustible, es decir, la capacidad de no sufrir combustión prematura o detonación a altos índices de compresión, en comparación a los de la gasolina (87) y el diésel (30). Otro aspecto importante es la energía mínima requerida para la ignición, la cual es de $0.017 \mathrm{~mJ}$ para el hidrógeno, valor por debajo de otros combustibles como el metano $(0.29 \mathrm{~mJ})$ y la gasolina $(0.24 \mathrm{~mJ})$. Otra de las ventajas de este combustible es su velocidad de quemado $(2.65 \mathrm{~m} / \mathrm{s}$ y $3.25 \mathrm{~m} / \mathrm{s})$, la cual indica que el hidrógeno se quema rápidamente, es decir, su combustión tiene poca duración [31].

Se pueden ver los beneficios del uso de tal combustible, aunque los procesos para su obtención y almacenamiento sean complicados y requieran mucha energía para realizarse. Dentro de las formas que se conocen de almacenamiento cabe destacar el almacenamiento como gas a presión, donde este es sometido en promedio a presiones de $200 \mathrm{~atm}$. Otra forma de almacenamiento es como líquido, en donde se requiere llevar el gas de hidrógeno a temperaturas entre los $14 \mathrm{~K}$ y $20 \mathrm{~K}$, mecanismo utilizado principalmente en la industria aeroespacial. Por último, se puede almacenar el hidrogeno en forma de hidruros metálicos en estado sólido [32], tecnología de almacenamiento que está en desarrollo, pero promete resultados muy buenos y relaciones de empaquetamiento considerablemente altas.

\subsection{Gas HHO (gas hidroxi)}

El gas hidroxi u oxihidrógeno es una mezcla de hidrógeno diatómico y oxígeno en una relación molar $2: 1$. Su proceso de combustión produce agua y $142.35 \mathrm{~kJ}$ de calor por cada gramo de hidrógeno. Dentro de sus propiedades se destacan su energía mínima de ignición, la cual está alrededor de los $0.02 \mathrm{~mJ}$, y su inflamabilidad en condiciones de temperatura de $298 \mathrm{~K}$ y presión de 1 atm en aire seco va desde $4 \%$ hasta un $94 \%$ de $\mathrm{HHO}$ [28][33]. Su método de obtención es mediante el proceso de electrólisis del agua.

El proceso de electrólisis para la obtención del gas HHO consiste en la descomposición de las moléculas de agua $\left(2 \mathrm{H}_{2} \mathrm{O}\right)$ en oxígeno $\left(\mathrm{O}_{2}\right)$ e hidrógeno $\left(2 \mathrm{H}_{2}\right)$, mediante la circulación de una corriente eléctrica en el agua. Para optimizar la producción del gas $\mathrm{HHO}$ es necesario modificar el $\mathrm{pH}$ del agua mediante un ácido o base, en este caso se utilizó hidróxido de sodio $(\mathrm{NaOH})$, ayudando así a la velocidad de la reacción.

La producción de gas está directamente relacionada con la corriente que circula por la celda, esto según la ley de la electrólisis de Faraday. El volumen producido de HHO se puede calcular utilizando la ecuación de Faraday para la electrólisis y la ecuación de los gases ideales:

$$
\begin{gathered}
m=\frac{E I t}{F} \\
p v=n R T
\end{gathered}
$$

donde $m$ representa la masa de la sustancia producida en el electrodo $(\mathrm{g}), E$ el peso equivalente $(\mathrm{g} / \mathrm{mol}), I$ la intensidad de corriente (A), $t$ el tiempo (s) y $F$ es la constante de Faraday $(96500 \mathrm{C} / \mathrm{mol}) . p$ representa la 
presión (atm), $v$ el volumen (L), $n$ es el número de moles, $R$ la constante universal de los gases $(0,082 \mathrm{~atm} \mathrm{~L} /$ $\left.\mathrm{mol}^{\circ} \mathrm{K}\right)$ y $\mathrm{T}$ la temperatura $\left({ }^{\circ} \mathrm{K}\right)$.

\section{DISEÑO DEL EXPERIMENTO}

Para las pruebas se instala el dispositivo de producción de gas HHO en el motor de una motocicleta de 4 tiempos marca Honda de 110CC, de encendido por chispa, con una potencia máxima de $8.6 \mathrm{HP}$ y 8000 RPM. Posteriormente, se miden las diferentes variables que se ven afectadas en el funcionamiento del motor al adicionar gas HHO, y se realiza una comparación con sus valores en condiciones donde solo se usa combustible tradicional.

\subsection{Configuración del sistema}

Este estudio se realizó utilizando el sistema propietario representado en la Figura 1, el cual consiste en una celda seca de HHO (1), conectada a una batería de $12 \mathrm{~V}$ (2), con un sistema de protección de sobrecargas (fusible) (3), la cual permite que se realice el proceso de electrólisis y se obtenga el gas. El gas realiza el recorrido hasta el tanque de almacenamiento del electrolito (4), que a su vez hace la función de burbujeador, de allí se dirige por una manguera hasta un filtro secador (5) que hace a su vez la función de válvula antiretorno de llama. Luego de este punto el gas es dirigido por otra manguera al filtro de aire de la moto (6), para ser suministrado en la cámara de combustión, entrando simultáneamente con el aire requerido para el proceso. De esta manera, se espera que el gas se mezcle con el aire y la gasolina en el carburador, y entren a la cámara de combustión para su encendido.

\subsection{Celda de HHO}

La celda consiste en un conjunto de placas octogonales de $41.4 \mathrm{~mm}$ de longitud en cada lado y espesor $1 \mathrm{~mm}$. Se utilizaron un total de 7 placas: una placa para polaridad positiva, otra placa para polaridad negativa y 5 placas neutras. Estas placas se fabricaron en acero inoxidable AISI 304 debido a que posee las propiedades necesarias para soportar la corrosión, producto de la reacción química que se presenta con el electrolito para la liberación de las moléculas de hidrógeno y oxígeno. Para los separadores entre placas se utilizaron anillos de neopreno de diámetro $90 \mathrm{~mm}$ y espesor $3 \mathrm{~mm}$. Las placas de soporte de la celda se fabricaron en acrílico, con un espesor considerable $(1 \mathrm{~cm})$ para que soporte las fuerzas aplicadas por las tuercas de sujeción y también para soportar algún imprevisto. Uno de los acrílicos posee dos perforaciones de $9.53 \mathrm{~mm}$, una para la circulación del agua proveniente del depósito y otra por donde sale el gas producido en la celda.

Para la conexión eléctrica se utilizó una batería de $12 \mathrm{~V}$ con un máximo de corriente de $3 \mathrm{~A}$, los cuales

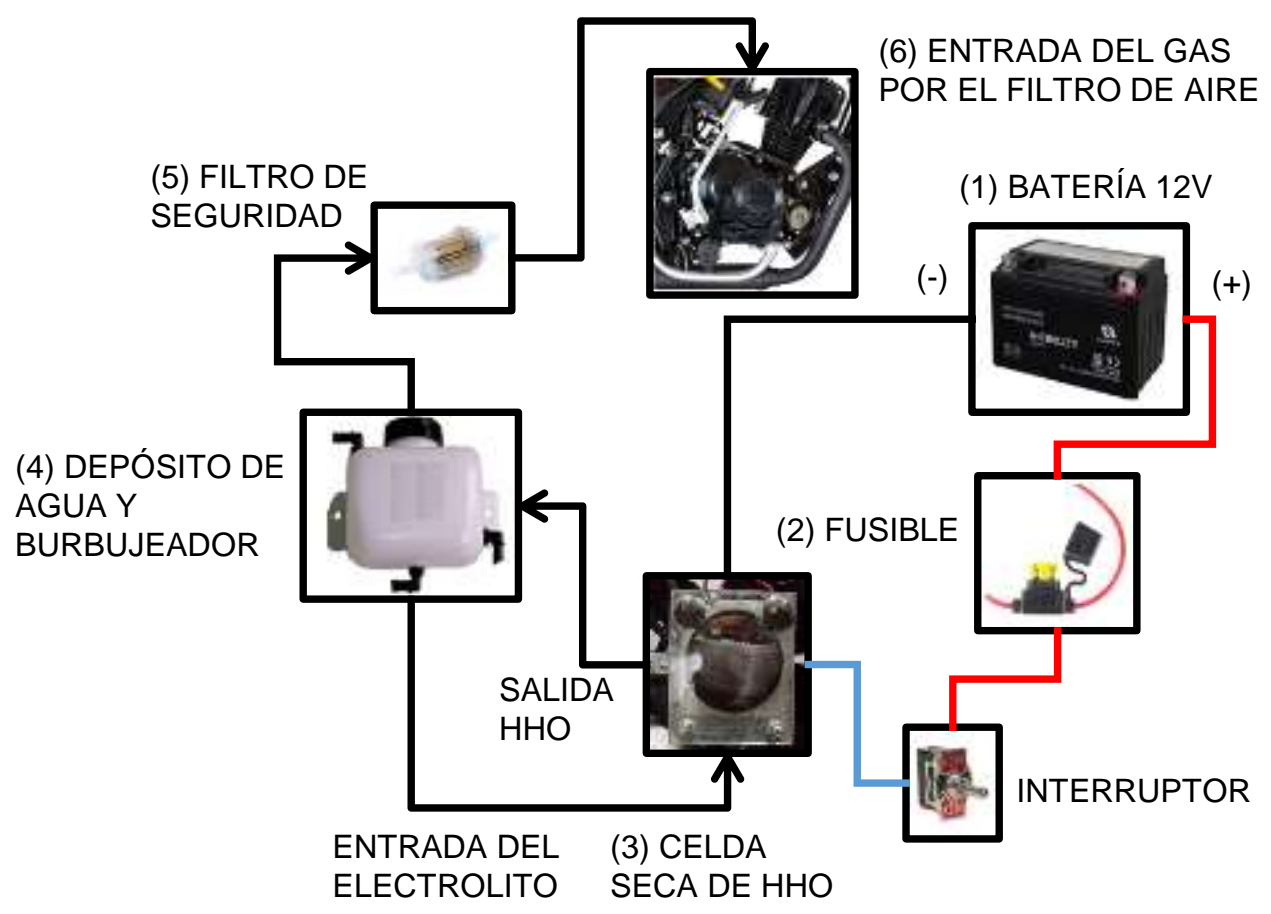

Figura 1. Esquema de montaje sistema de producción de HHO. 
permanecen aproximadamente constantes en la utilización del dispositivo siempre y cuando la motocicleta este encendida. Se instaló un sistema de seguridad para las sobrecargas o corto circuito, el cual consta de un fusible de capacidad máxima de $10 \mathrm{~A}$ y un interruptor para el encendido y apagado de la celda. Para las condiciones de operación de $0.9 \mathrm{~atm}, 31.4^{\circ} \mathrm{C}$ y $3 \mathrm{~A}$, se alcanza una producción de $\mathrm{HHO}$ de $0.19 \mathrm{~L} / \mathrm{min}$.

Para la instalación en la motocicleta, la celda se dispuso de manera que quede posicionada en un lado de la motocicleta y el tanque de depósito-burbujeador quede lo más alto posible respecto a la celda, Figura 2 esto con el fin de facilitar el movimiento del electrolito por gravedad para el llenado del dispositivo, ya que este debe estar, en lo posible, completamente lleno de líquido, para así aprovechar el área de contacto de las placas de acero con el electrolito.

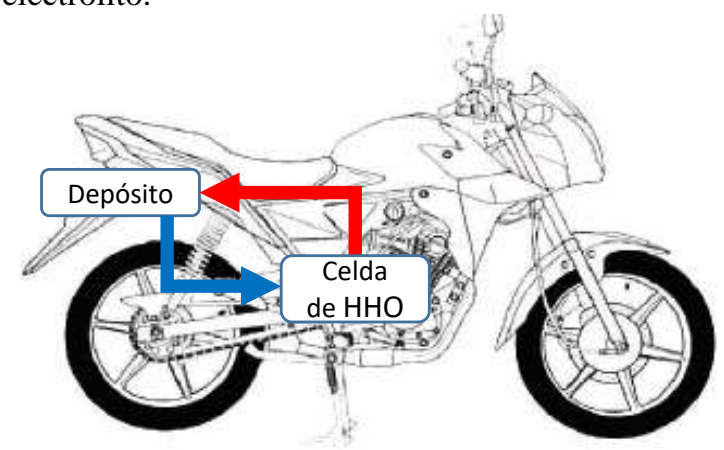

Figura 2. Posición del dispositivo en la motocicleta.

\section{RESULTADOS}

\subsection{Prueba de consumo de combustible}

Para la prueba de consumo de combustible es posible utilizar métodos volumétricos o gravimétricos. En este trabajo se utilizó un método volumétrico, para lo cual se fabricó un elemento que permitiera la medición de la cantidad de gasolina que se suministra al carburador (Figura 3). Esto se hizo con el fin de poder medir el tiempo transcurrido en el consumo total del combustible en el depósito a 5000, 6000 y 7000 RPM, utilizando un medidor de revoluciones MGT 300 EVO. Se promediaron los tiempos de 10 pruebas en cada una de los valores de RPM establecidos. En la Figura 4 se muestra la conexión del dispositivo de dosificación al carburador.

En la Figura 5 se representan los resultados de la prueba de consumo comparando los resultados con y sin adición de HHO. En esta gráfica se observa el flujo volumétrico $(\mathrm{mL} / \mathrm{s})$ de la motocicleta adicionando gas HHO y sin él, para valores de 5000 RPM, 6000 RPM y 7000 RPM. Se ha tomado este intervalo de RPM puesto que, a las 6000 RPM, aproximadamente, se presenta el mayor torque ofrecido por el motor según datos del fabricante. Para el consumo de combustible dado por el flujo volumétrico se observó un ligero incremento para la gasolina respecto a la mezcla gasolina-HHO. Se tuvo una disminución en el flujo volumétrico del $8.4 \%$ para cuando al mezclar gas HHO en la combustión con gasolina.

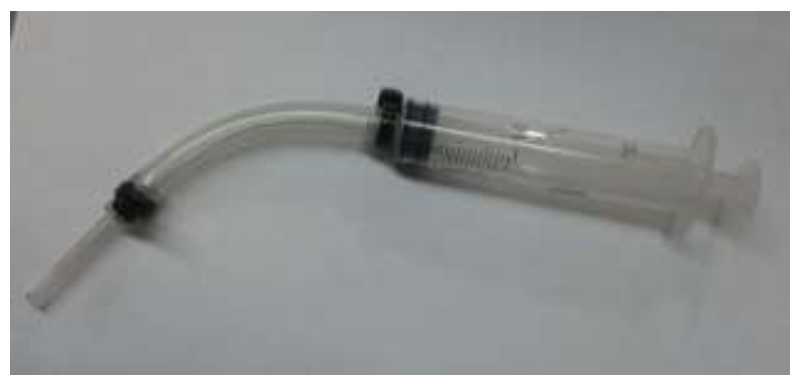

Figura 3. Dispositivo para dosificación del combustible.

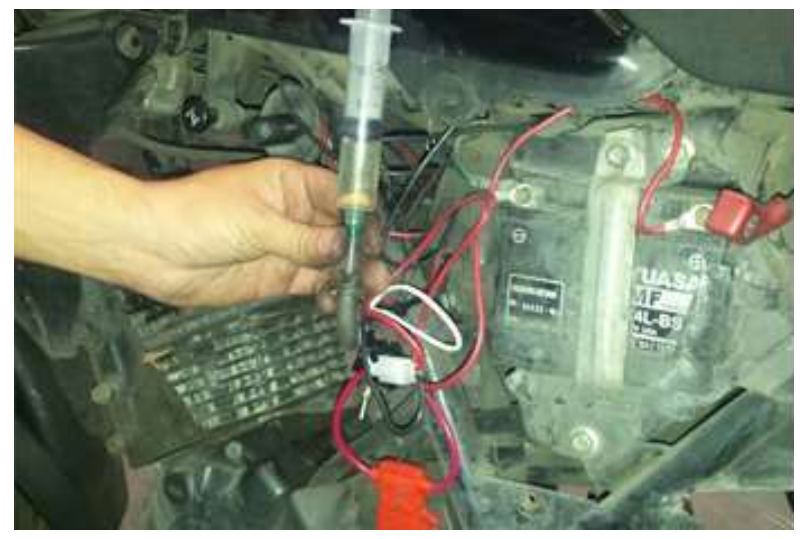

Figura 4. Montaje del dispositivo dosificador. Conexión de la manguera dosificadora al carburador.

Dicha ventaja podría ser mayor implementando dispositivos electrónicos que permitan controlar el ingreso del gas $\mathrm{HHO}$ a la mezcla de combustible y el aire, manteniendo así una mezcla estequiométrica que permita que se lleve a cabo una combustión completa. Esto permitiría determinar el volumen de gas administrado que reemplaza parte del combustible tradicional, disminuyendo el consumo de gasolina. Un dispositivo de dosificación del HHO llamado HECU (unidad de control electrónico de hidroxi) se utilizó en [21], el cual cumplió la función de regular la salida del gas HHO mediante el control del voltaje y la corriente que llega a la celda, para obtener eficiencias mayores en la combustión de motores de encendido por compresión. 


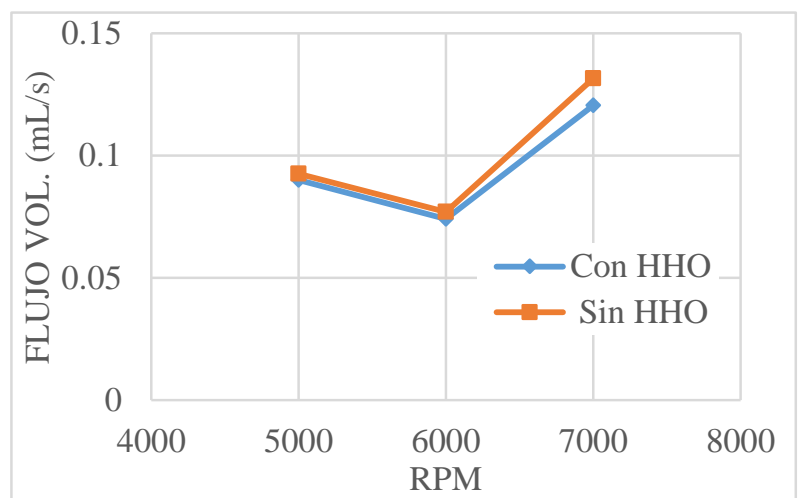

Figura 5. Comportamiento del consumo de combustible respecto a las RPM del motor con y sin adición de gas HHO.

\subsection{Prueba de eficiencia mecánica}

Al tener el dispositivo instalado y funcionando se procede a realizar en primer lugar el análisis de la eficiencia mecánica $\eta$ dada por:

$$
\eta=\frac{\text { potencia experimental }}{\text { potencia teórica }}
$$

donde la potencia experimental es medida mediante el uso de un dinamómetro inercial de rodillos con una capacidad máxima de medición de 25 HP [34], que permite saber la potencia del motor en funcionamiento con solo gasolina y con la mezcla de HHO-gasolina. La eficiencia del motor se calculó para ambos casos, haciendo la comparación respectiva entre los resultados. Se trabajó en función del tiempo la variación de las potencias medidas, para hacer así las gráficas comparativas del comportamiento de la potencia en el tiempo transcurrido y con la variación de las RPM del motor, llevándolo hasta el punto de potencia máxima. Esta prueba se realizó en la tercera marcha de la motocicleta.

La Figura 6 muestra los resultados obtenidos de la variación de la potencia respecto al tiempo para ambos casos: suministro de combustible puro y con HHO. Se observa como la potencia aumenta hasta alcanzar su punto máximo. La potencia alcanza sus valores máximos con el sistema HHO en un tiempo de $13 \mathrm{~s}$ aproximadamente, tomando como valor máximo de potencia alcanzada 7.54 HP. Para el sistema sin HHO la potencia máxima es de $7.605 \mathrm{HP}$ y se alcanza en $12 \mathrm{~s}$ aproximadamente. Aunque la diferencia es mínima para los dos casos, se puede decir que en lo que respecta a la potencia máxima respecto al tiempo, el sistema sin $\mathrm{HHO}$ sería un $7.7 \%$ más rápido en alcanzar la potencia máxima que el sistema con HHO, además de alcanzar un $0.855 \%$ más de potencia en relación con el sistema HHO. Este comportamiento es de esperarse ya que no se han realizado modificaciones al avance de la chispa ni a la regulación del carburador. Como se observa en la literatura [27], resulta necesario ajustar dichos parámetros para la optimización del desempeño del motor híbrido.

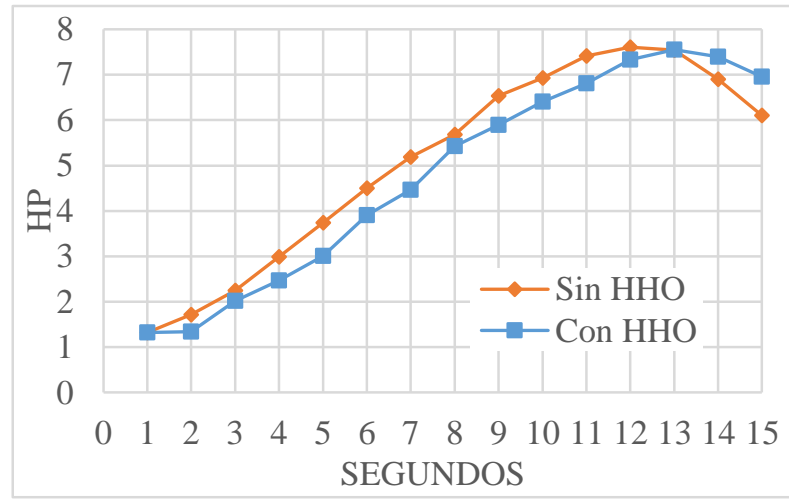

Figura 6. Potencia vs Tiempo.

En la Figura 7 se observa como el sistema con gas $\mathrm{HHO}$ alcanza su valor máximo de potencia $7.54 \mathrm{HP}$ a 7700 RPM, comparado con el sistema sin HHO, que alcanza $7.605 \mathrm{HP}$ a las 7800 RPM. Estos resultados ayudan a complementar el análisis anterior con respecto al tiempo, dado que se producen mayores RPM en menor tiempo sin la adición del gas a la mezcla. No obstante, se observa que al momento de alcanzar la potencia máxima en ambos casos (con y sin HHO), esta empieza a disminuir, mostrando como la potencia de la mezcla sin HHO sufre una disminución mayor que para el gas HHO.

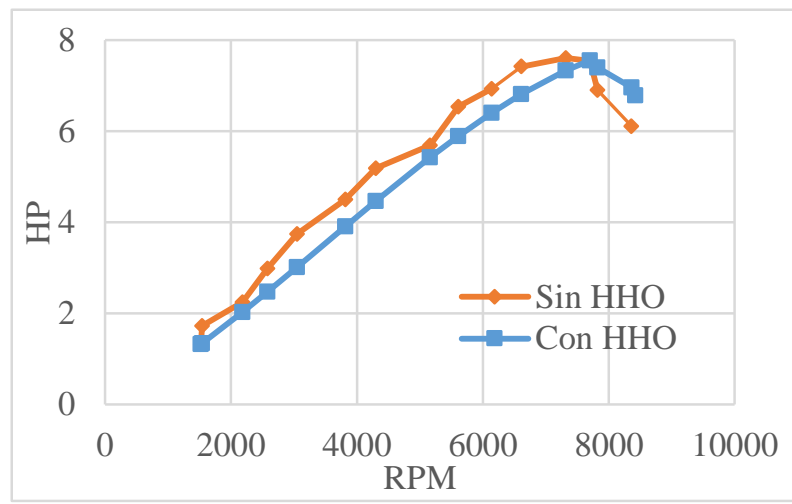

Figura 7. Potencia vs RPM.

En la Figura 8 se observa que el motor alcanza su valor máximo de 8.98 N.m a 6290 RPM sin adicionar gas HHO a la mezcla de combustión, en comparación con 8.96 N.m a 6140 RPM con HHO. La Figura 8 también muestra 
como el torque disminuye más rápidamente en el caso de la mezcla de combustión sin HHO después de alcázar su torque máximo. Lo que lleva a concluir que el uso del sistema HHO disminuye el torque que entrega el motor de inicio a fin de la prueba.

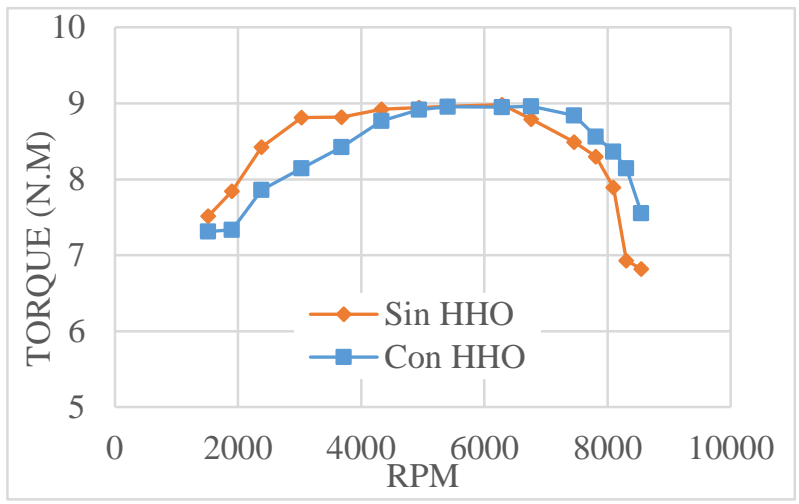

Figura 8. Torque vs RPM.

En la Figura 9 se observa que la variación de las RPM es proporcional al tiempo, dado que a medida que avanza el mismo se va alcanzado un mayor número de revoluciones. Para el sistema HHO se alcanzan 8360 RPM en $15 \mathrm{~s}$, y para el sistema HHO se alcanzan 8550 RPM en $15 \mathrm{~s}$.

Estos valores sugieren que, aunque son valores muy cercanos, el sistema HHO presenta una menor eficiencia, con un $87 \%$, en contraste con un $88 \%$ de eficiencia sin el sistema aplicado. Cabe resaltar que estos datos fueron obtenidos con un margen de error, debido a los dispositivos de medición usados.

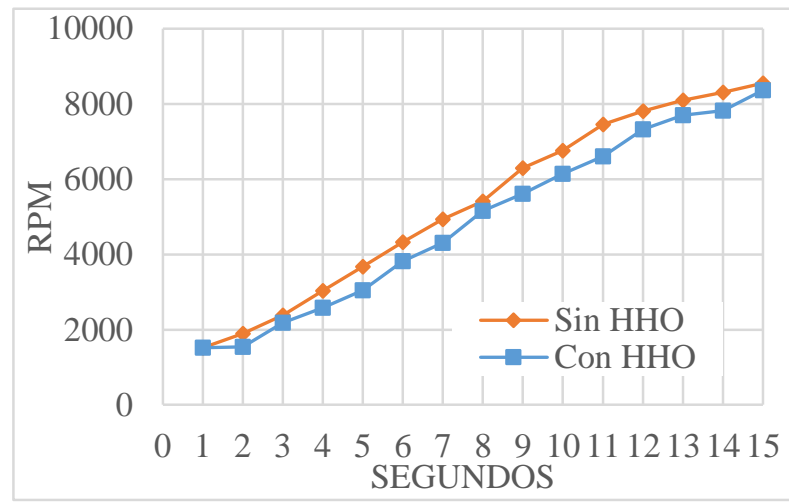

Figura 9. RPM vs Tiempo.

En la Tabla 2 se muestran las eficiencias calculadas para ambos casos, donde se toma como potencia teórica, la potencia nominal que viene especificada por la empresa fabricante, y la potencia experimental, la que se obtiene de las pruebas.

Tabla 2. Potencia (HP) y eficiencia mecánica con y sin gas HHO.

\begin{tabular}{|l|l|l|l|}
\hline & $\begin{array}{l}\text { Potencia } \\
\text { teórica }\end{array}$ & $\begin{array}{l}\text { Potencia } \\
\text { experimental }\end{array}$ & Eficiencia \\
\hline Sin HHO & 8.6 & 7.605 & 0.884 \\
\hline Con HHO & 8.6 & 7.550 & 0.878 \\
\hline
\end{tabular}

\subsection{Prueba de gases contaminantes}

Esta prueba se realizó con el fin de identificar las posibles variaciones en las cantidades de los gases emitidos por la combustión, de tal manera que se pudieran identificar y comparar estos productos al utilizar el combustible tradicional (gasolina) y la mezcla de HHO-gasolina. Para esta prueba se utilizó un dispositivo MGT 300 EVO para la medición de las RPM y un analizador de gases JB-SPI (Figura 10) el cual está diseñado para medir la concentración de gases en una muestra de humos de combustión, bajo el método de infrarrojo no dispersivo (NDIR) y celda electroquímica para la medición de O2. Este dispositivo toma lecturas de gases como $\mathrm{HC}$ (hidrocarburos), que corresponde a la gasolina cruda que no se quema, CO (monóxido de carbono), que corresponde a gasolina mal quemada y contaminante, $\mathrm{CO}_{2}$ (dióxido de carbono) de la gasolina quemada en el punto óptimo de sincronización y $\mathrm{O}_{2}$ (oxígeno).

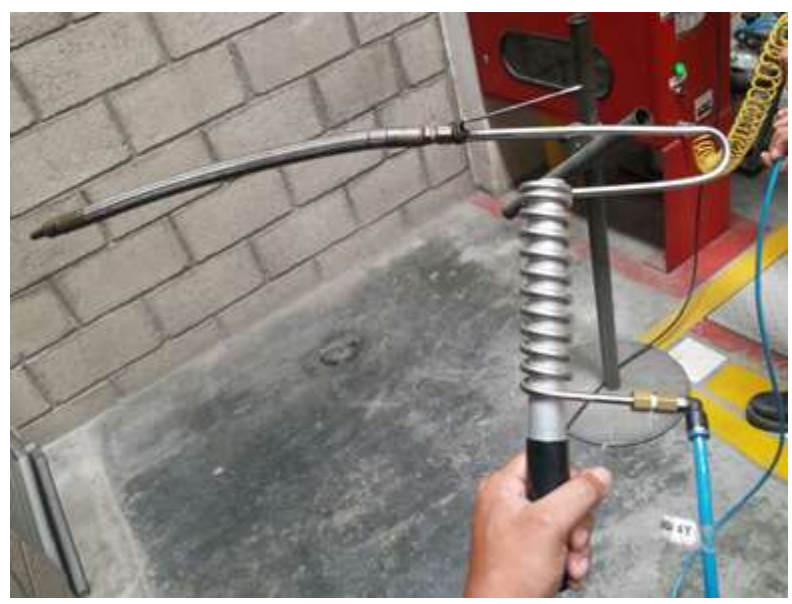

Figura 10. Analizador de gases JB-SPI.

Para la prueba, se realizó el montaje del sensor en la motocicleta (Figura11) de manera que captara la mayor cantidad de gases del tubo de escape, se midieron los porcentajes volumétricos de los gases contaminantes respecto a la variación de las RPM del motor, y las parte por millón (ppm) de los HC también respecto a las RPM. 


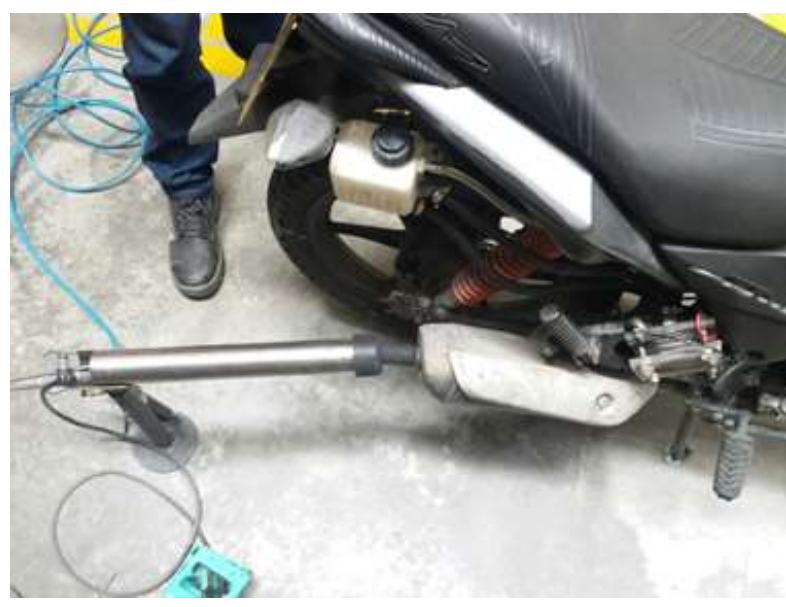

Figura 11. Montaje de sensor JB-SPI en el tubo de escape de la motocicleta.

En la Figura 12 se observa que las emisiones contaminantes provenientes del monóxido de carbono (CO) disminuyen al agregar el gas HHO. Con el aumento de las RPM del motor, se presenta una disminución de las emisiones de un $13 \%$ al $18 \%$.

En la Figura 13 se representa el incremento de $\mathrm{CO}_{2}$ con la adición de gas $\mathrm{HHO}$, mostrando con esto una mejora en la gasolina que se quema en el punto óptimo de sincronización. Para el dióxido de carbono $\left(\mathrm{CO}_{2}\right)$ se apreció un incremento que va desde un $5 \%$ a un $9 \%$ en volumen de $\mathrm{CO}_{2}$

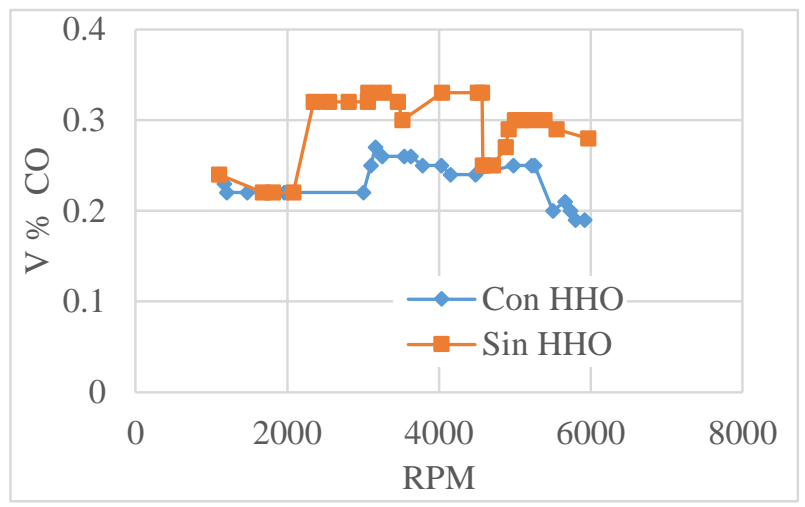

Figura 12. Porcentaje de CO vs RPM.

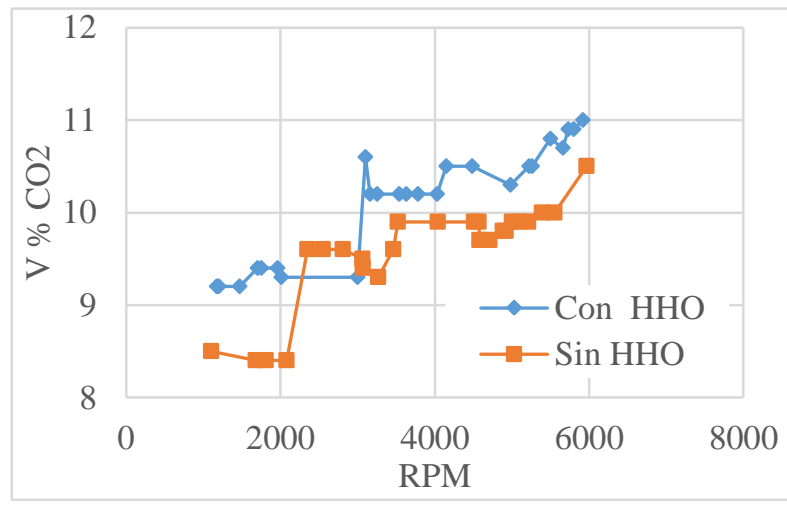

Figura 13. Porcentaje de $\mathrm{CO}_{2}$ vs RPM.

En la Figura 14 se representa la disminución de la cantidad de oxígeno en la mezcla cuando esta tiene el gas $\mathrm{HHO}$, en comparación con la gasolina sola. Esto es debido a que se ha utilizado un volumen mayor de $\mathrm{O}_{2}$ en la combustión para la producción de $\mathrm{CO}_{2}$. La disminución registrada está entre un $19 \%$ y $20 \%$ en volumen de $\mathrm{O}_{2}$.

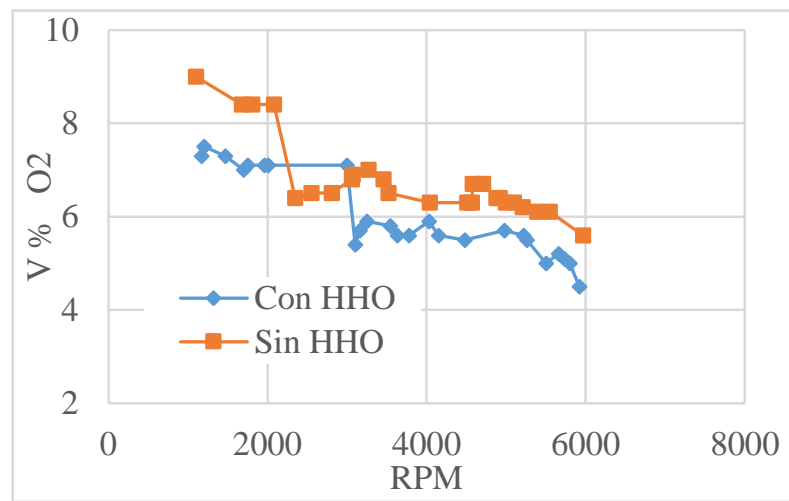

Figura 14. Porcentaje de $\mathrm{O}_{2}$ vs RPM.

Por último, en la Figura 15 se muestra las ppm de los hidrocarburos (HC). Se ve también una disminución para la combustión con $\mathrm{HHO}$, en comparación con la combustión de solo gasolina. Es decir, se están quemando mayores proporciones de combustible y no están saliendo crudas al ambiente. Las emisiones bajaron en un rango del $30 \%$ al $54 \%$, de acuerdo a la variación de las RPM del motor entre 1100 RPM y 7000 RPM. 
152 REVISTA UIS

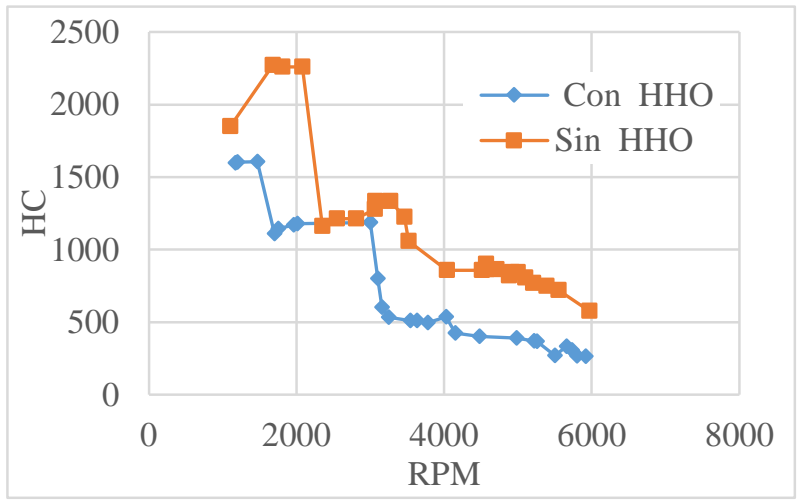

Figura 15. Partes por millón de HC vs RPM.

\subsection{Consumo específico de combustible}

Se ha calculado el consumo específico de combustible, SFC, el cual indica la eficiencia que tiene un motor para transformar combustible en energía mecánica, y se expresa como la cantidad de combustible que hay que consumir para obtener una determinada potencia, durante una hora $(\mathrm{g} / \mathrm{kWh})$ [35]. Para esto se usaron las siguientes ecuaciones:

$$
\begin{gathered}
\mathrm{SFC}=\frac{m_{f}}{P b}, \\
m_{f}=\frac{\Delta m_{T f t}}{\Delta t}, \\
\Delta m_{T f t}=\frac{V t_{l t} \rho_{\text {aire }} n c_{h} t}{3600 \mathrm{rel}_{a / c}},
\end{gathered}
$$

donde SFC es el consumo específico de combustible en $\mathrm{g} /(\mathrm{kWh}), m_{f}$ representa el flujo másico de combustible (g/h) y $P b$ es la potencia de freno (KW). Para el cálculo del flujo másico se evalúa la masa total de combustible $\Delta m_{T f t}$ en un tiempo $\Delta t$. Para el cálculo de la masa, $V t_{l t}$ representa el volumen del cilindraje de la motocicleta en litros, $\rho_{\text {aire }}$ la densidad del aire $(\mathrm{g} / \mathrm{L}), n c_{h}$ el número de ciclos por hora a determinadas RPM, y $\mathrm{rel}_{a / c}$ la relación aire combustible para una mezcla rica, que para este caso se toma de 13.5.

En la Figura16 muestra la variación del SFC respecto a las RPM del motor, en donde se puede apreciar una disminución del SFC aproximadamente de un $7.3 \%$ al adicionar el gas $\mathrm{HHO}$, en comparación cuando este no se utiliza.
A. O. Diaz-Rey, J. E. González-Gil, O. A. González-Estrada

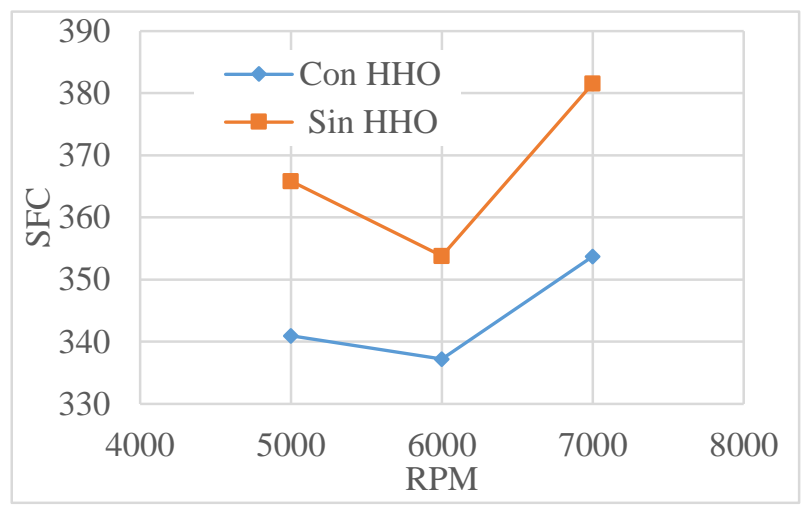

Figura 16. SFC VS RPM.

\section{CONCLUSIONES}

Se implementó un sistema de HHO en un motor de 110 $\mathrm{cm}^{3}$. Se registró el consumo de combustible en la motocicleta variando respecto a las RPM, considerando la utilización o no de gas $\mathrm{HHO}$ en la mezcla de la combustión. Se mostró que el tiempo que requiere el motor para consumir $10 \mathrm{~mL}$ de gasolina tuvo un ligero incremento en las pruebas donde se adicionó el gas HHO, siendo así que a 5000 RPM el tiempo aumento en $2.7 \%$, para 6000 RPM incrementó un $3.7 \%$ y para 7000 RPM un valor de $8.5 \%$ en el tiempo de consumo. Por lo tanto, el dispositivo puede ayudar a la disminución del consumo de gasolina, aunque en esta prueba las diferencias no hayan sido muy significativas.

En cuanto a la eficiencia obtenida para el motor de 110 $\mathrm{cm}^{3}$ con la adición de gas HHO a la mezcla de combustión, se registraron valores de 0.87 , y sin la adición del gas a la mezcla de 0.88 . Estos valores indican una diferencia muy mínima, y que el sistema HHO disminuye ligeramente la eficiencia mecánica del motor. Se obtuvieron los valores de HP máximos alcanzados por el experimento, dando como resultado $7.605 \mathrm{HP}$ a 7800 RPM sin el sistema de HHO, y 7.54 HP a 7700 RPM en un tiempo de 12 s y 13 s, respectivamente. Los resultados sin el sistema $\mathrm{HHO}$ son ligeramente mejores en relación a la potencia suministrada por el motor, obteniéndose como resultado un $88 \%$, en contraste con un $87 \%$ de eficiencia obtenida con el sistema HHO. Mediante el análisis gráfico se mostró como la potencia y el torque disminuyen después de alcanzar sus puntos máximos más rápido en el sistema sin $\mathrm{HHO}$.

En la prueba de gases se observaron mejoras importantes, indicando altos beneficios de la mezcla de gas HHO gasolina para el proceso de combustión, en comparación a la combustión solo con la gasolina. Estos resultados fueron: una disminución en las emisiones de monóxido de carbono (CO) del 13\% al 18\%, el cual es un gas tóxico 
y muy contaminante. Para el dióxido de carbono $\left(\mathrm{CO}_{2}\right)$ se apreció un incremento que del $5 \%$ al 9\% con gas HHO, mostrando una mejora en la quema del combustible. Las emisiones de oxigeno $\left(\mathrm{O}_{2}\right)$ disminuyeron en un $20 \%$, indicando que el gas $\mathrm{HHO}$ ha enriquecido la mezcla en la cámara de combustión, aprovechando más oxígeno para su combustión. Por último, se observó la reducción en las ppm de los hidrocarburos (HC) entre un $30 \%$ a un 54\%, resultado de que se enriquece la mezcla y se quema más combustible. Los hidrocarburos sin quemar reducen su cantidad al salir por el tubo de escape cuando se utiliza el gas HHO. En general, las ventajas de la utilización de este gas en la combustión para la disminución de emisiones nocivas son apreciables.

Se obtuvo una disminución en el valor del consumo específico de combustible, SFC, de $7.3 \%$ al ser adicionado a la mezcla gas $\mathrm{HHO}$, haciendo un análisis a 5000, 6000 y 7000 RPM, lo que corrobora que este sistema disminuye el SFC.

Como trabajos futuros se propone un estudio más detallado de las variaciones en la inyección de la mezcla y el ajuste del avance de la chispa, con el fin de optimizar el proceso de combustión. También resulta necesario realizar un análisis del consumo eléctrico de la celda y el balance energético del sistema completo.

\section{REFERENCIAS}

[1] V. Álvarez-Narvaez, E. Quiñones-Bolaños, M. E. Huertas-Bolaños, C. A. Suárez, J. Berdugo-Arrieta, and D. Ramírez-Rivas, "Metodología para la selección de sitios de monitoreo atmosférico en zonas urbanas afectada por las emisiones de fuentes móviles," Rev. UIS Ing., vol. 15, no. 2, pp. 73-84, 2016.

[2] P. P. Vergara-Barrios, J. M. Rey-López, G. A. Osma-Pinto, and G. Ordoñez-Plata, "Evaluación del potencial solar y eólico del campus centra de la Universidad Industrial de Santander y la ciudad de Bucaramanga, Colombia," Rev. UIS Ing., vol. 13, no. 2, pp. 49-57, 2014.

[3] Y. J. Rueda Ordóñez and K. K. Tannous, "Análisis cinético de la descomposición térmica de biomasas aplicando un esquema de reacciones paralelas independientes," Rev. UIS Ing., vol. 16, no. 2, pp. 119128, 2017.

[4] A. Boretti, "Comparison of fuel economies of high efficiency diesel and hydrogen engines powering a compact car with a flywheel based kinetic energy recovery systems," Int. J. Hydrogen Energy, vol. 35, no. 16, pp. 8417-8424, Aug. 2010.
[5] C. M. White, R. R. Steeper, and A. E. Lutz, "The hydrogen-fueled internal combustion engine: a technical review," Int. J. Hydrogen Energy, vol. 31, no. 10, pp. 1292-1305, Aug. 2006.

[6] V. Knop, A. Benkenida, S. Jay, and O. Colin, "Modelling of combustion and nitrogen oxide formation in hydrogen-fuelled internal combustion engines within a 3D CFD code," Int. J. Hydrogen Energy, vol. 33, no. 19, pp. 5083-5097, Oct. 2008.

[7] M. Momirlan and T. N. Veziroglu, "The properties of hydrogen as fuel tomorrow in sustainable energy system for a cleaner planet," Int. J. Hydrogen Energy, vol. 30, no. 7, pp. 795-802, Jul. 2005.

[8] N. Saravanan and G. Nagarajan, "An experimental investigation on optimized manifold injection in a directinjection diesel engine with various hydrogen flowrates," Proc. Inst. Mech. Eng. Part D J. Automob. Eng., vol. 221, no. 12, pp. 1575-1584, Dec. 2007.

[9] N. Saravanan, G. Nagarajan, C. Dhanasekaran, and K. M. Kalaiselvan, "Experimental investigation of hydrogen port fuel injection in DI diesel engine," Int. $J$. Hydrogen Energy, vol. 32, no. 16, pp. 4071-4080, Nov. 2007.

[10] N. Saravanan, G. Nagarajan, K. M. Kalaiselvan, and C. Dhanasekaran, "An experimental investigation on hydrogen as a dual fuel for diesel engine system with exhaust gas recirculation technique," Renew. Energy, vol. 33, no. 3, pp. 422-427, Mar. 2008.

[11] S. Szwaja and K. Grab-Rogalinski, "Hydrogen combustion in a compression ignition diesel engine," Int. J. Hydrogen Energy, vol. 34, no. 10, pp. 4413-4421, May 2009.

[12] M. Masood, M. M. Ishrat, and A. S. Reddy, "Computational combustion and emission analysis of hydrogen-diesel blends with experimental verification," Int. J. Hydrogen Energy, vol. 32, no. 13, pp. 2539-2547, Sep. 2007.

[13] M. K. Baltacioglu, H. T. Arat, M. Özcanli, and K. Aydin, "Experimental comparison of pure hydrogen and HHO (hydroxy) enriched biodiesel (B10) fuel in a commercial diesel engine," Int. J. Hydrogen Energy, vol. 41, no. 19, pp. 8347-8353, May 2016.

[14] S. O. Akansu, Z. Dulger, N. Kahraman, and T. N. Veziroğlu, "Internal combustion engines fueled by natural gas-hydrogen mixtures," Int. J. Hydrogen Energy, vol. 29, no. 14, pp. 1527-1539, Nov. 2004. 
[15] F. Ma et al., "Performance and emission characteristics of a turbocharged spark-ignition hydrogen-enriched compressed natural gas engine under wide open throttle operating conditions," Int. J. Hydrogen Energy, vol. 35, no. 22, pp. 12502-12509, Nov. 2010.

[16] C. Ji, B. Zhang, and S. Wang, "Enhancing the performance of a spark-ignition methanol engine with hydrogen addition," Int. J. Hydrogen Energy, vol. 38, no. 18, pp. 7490-7498, Jun. 2013.

[17] T. D'Andrea, P. F. Henshaw, and D. S.-K. Ting, "The addition of hydrogen to a gasoline-fuelled SI engine," Int. J. Hydrogen Energy, vol. 29, no. 14, pp. 1541-1552, Nov. 2004.

[18] F. Ma and Y. Wang, "Study on the extension of lean operation limit through hydrogen enrichment in a natural gas spark-ignition engine," Int. J. Hydrogen Energy, vol. 33, no. 4, pp. 1416-1424, 2008.

[19] S. A. Musmar and A. A. Al-Rousan, "Effect of HHO gas on combustion emissions in gasoline engines," Fuel, vol. 90, no. 10, pp. 3066-3070, Oct. 2011.

[20] A. A. Al-Rousan, "Reduction of fuel consumption in gasoline engines by introducing $\mathrm{HHO}$ gas into intake manifold," Int. J. Hydrogen Energy, vol. 35, no. 23, pp. 12930-12935, Dec. 2010.

[21] A. C. Yilmaz, E. Uludamar, and K. Aydin, "Effect of hydroxy (HHO) gas addition on performance and exhaust emissions in compression ignition engines," Int. J. Hydrogen Energy, vol. 35, no. 20, pp. 11366-11372, Oct. 2010.

[22] K. V Shivaprasad, S. Raviteja, P. Chitragar, and G. N. Kumar, "Experimental Investigation of the Effect of Hydrogen Addition on Combustion Performance and Emissions Characteristics of a Spark Ignition High Speed Gasoline Engine," Procedia Technol., vol. 14, pp. 141148, 2014.

[23] S. Wang, C. Ji, J. Zhang, and B. Zhang, "Improving the performance of a gasoline engine with the addition of hydrogen-oxygen mixtures," Int. J. Hydrogen Energy, vol. 36, no. 17, pp. 11164-11173, Aug. 2011.

[24] S. Wang, C. Ji, J. Zhang, and B. Zhang, "Comparison of the performance of a spark-ignited gasoline engine blended with hydrogen and hydrogenoxygen mixtures," Energy, vol. 36, no. 10, pp. 58325837, Oct. 2011.

[25] S. Wang and C. Ji, "Cyclic variation in a hydrogen- enriched spark-ignition gasoline engine under various operating conditions," Int. J. Hydrogen Energy, vol. 37, no. 1, pp. 1112-1119, Jan. 2012.

[26] C. Ji and S. Wang, "Effect of hydrogen addition on combustion and emissions performance of a spark ignition gasoline engine at lean conditions," Int. J. Hydrogen Energy, vol. 34, no. 18, pp. 7823-7834, Sep. 2009.

[27] C. Ji, S. Wang, and B. Zhang, "Effect of spark timing on the performance of a hybrid hydrogengasoline engine at lean conditions," Int. J. Hydrogen Energy, vol. 35, no. 5, pp. 2203-2212, Mar. 2010.

[28] E. M. Quezada Romero, D. F. Torres Gualan, Q. Romero, E. Miguel, T. Gualan, and D. Fernando, "Implementación de un generador de hidrógeno de celda seca en un vehículo Chevrolet Steem 1.6 L," Universidad politecnica salesiana, 2014.

[29] D. González, Motores térmicos y sus sistemas auxiliares, Segunda Ed. Madrid, 2015.

[30] Xunta de Galicia, "Análisis de gases." [Online]. Available:

http://www.edu.xunta.gal/centros/cifpsomeso/system/fil es/ANALISIS+DE+GASES.pdf. [Accessed: 25-Mar2017].

[31] L. Gutiérrez Jodra, "El Hidrógeno, Combustible Del Futuro," Cienc.Exact.Fís.Nat. (Esp), vol. 99, no. 1, pp. 49-67, 2005.

[32] B. Choi, D. Panthi, M. Nakoji, T. Kabutomori, K. Tsutsumi, and A. Tsutsumi, "Novel hydrogen production and power generation system using metal hydride," Int. J. Hydrogen Energy, vol. 40, no. 18, pp. 6197-6206, 2015.

[33] H. F. Coward, G. W. Jones, Etats-Unis, and Mines (Bureau), "Limits of flammability of gases and vapors, by H. F. Coward and G. W. Jones.," Industrial \& Engineering Chemistry Research, vol. 52, no. 17. pp. 6057-6067, 1952.

[34] E. Hormiga and E. Moreno, "Diseño y construcción de un dinamómetro de motos para la empresa JB Soluciones Prácticas de Ingeniería," Universidad Industrial de Santander, 2013.

[35] J. B. Heywood, Internal Combustion Engine Fundamentals, 3ra ed. Michigan: McGraw-Hill, 1988. 